\title{
Analysis of temperature-induced drift rate error in interferometric multi-core fiber optic gyroscope
}

Shinji Mitani, Tadahito Mizutani, Yusaku Tottori, Haruyuki Endo, Yuichi Takushima

Shinji Mitani, Tadahito Mizutani, Yusaku Tottori, Haruyuki Endo, Yuichi Takushima, "Analysis of temperature-induced drift rate error in interferometric multi-core fiber optic gyroscope," Proc. SPIE 11852, International Conference on Space Optics - ICSO 2020, 118523E (11 June 2021); doi: 10.1117/12.2599545

SPIE Event: International Conference on Space Optics - ICSO 2021, 2021, Online Only 


\section{International Conference on Space Optics-ICSO 2020}

Virtual Conference

30 March-2 April 2021

Edited by Bruno Cugny, Zoran Sodnik, and Nikos Karafolas
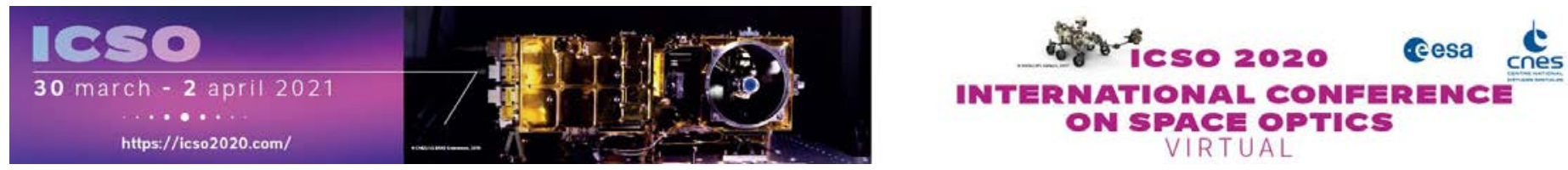

\section{Analysis of temperature-induced drift rate error in interferometric multi-core fiber optic gyroscope}

\section{Cesa isoporecestings denes}




\title{
Analysis of temperature-induced drift rate error in Interferometric multi-core fiber optic gyroscope
}

\author{
Shinji Mitani*a, Tadahito Mizutani ${ }^{\mathrm{a}}$, Yusaku Tottori ${ }^{\mathrm{b}}$, Haruyuki Endo ${ }^{\mathrm{b}}$, Yuichi Takushima ${ }^{\mathrm{b}}$ \\ aJapan Aerospace Exploration Agency (JAXA), 2-1-1 Sengen, Tsukuba, Ibaraki, Japan 305-8505; \\ ${ }^{\mathrm{b}}$ Optoquest Company, Ltd., 1335 Haraichi, Ageo, Saitama, Japan 362-0021
}

\begin{abstract}
This paper applies non-reciprocal phase error analysis approach to the fiber optic gyroscope coil made of multi-core fiber and fan-in/fan-out devices to estimate the thermal sensitivity analytically for the first time. In the case of MCF symmetric coils, the relationship between the coil parameters, which can take advantage of MC-FOG in terms of sensitivity to temperature change, is quantitatively clarified in comparison with SMF symmetric coils. In addition, the non-reciprocal phase error of the FIFO element under temperature disturbance is quantitatively clarified. The design policy of the coils proposed from these results is also discussed. And method for connecting cores that minimizes the phase error is proposed. As analytical results, besides lowering the manufacturing cost of the coil due to the shorter fiber length, the multi-core fiber coil was found to further reduce the phase error sensitivity compared to a single-mode coil of equivalent length.
\end{abstract}

Keywords: interferometric fiber-optic gyroscope, Sagnac effect, multi-core fiber, fan-in/fan-out, non-reciprocal phase error

\section{INTRODUCTION}

\subsection{Background}

Improving the sensitivity of the interferometric fiber-optic gyroscope (I-FOG) as a rotation-rate sensor contributes to usher in a new era of highly precise and autonomous mobile systems in space, marine and other applications. One solution for such sensitivity improvement is applying long fiber in the sensing coil. However, longer sensing fiber loop causes other problems. A non-reciprocal phase shift error [1] becomes relatively high compared to the reduced short-term noise and the unique manufacturing process of winding called symmetry winding [2] is inevitable. Symmetry winding process must require perfect qualification without coil turn number error or winding flaw. Therefore, the unique manufacturing process of winding such a long length of fiber into multiple layers to form a coil requires elaborate and costly skill. In the literature [3], we demonstrated a new interferometric fiber optic gyro (I-FOG) using multi-core fiber (MCF) and fan-in/fan-out (FIFO) devices, which pass through the core waveguide of MCF for N cores to form one large interference loop. One of the clear advantages of using MCF is that it allows us to drastically reduce the physical fiber length while maintaining the gyro's sensitivity. Since the number of turns of the fiber coil becomes $1 / \mathrm{N}$, the cost of the winding process can be also drastically reduced. Moreover, due to the small core-pitch of MCF, many core waveguides are densely packed in the coil. This results in the fiber coil being very compact, thereby raising expectations that the influence of the non-reciprocal phase error (Shupe effect) can be suppressed effectively. The proposed fiber coils were fabricated using a $102 \mathrm{~m}$-long 7-core MCF and implemented in an open-loop I-FOG system [3]. We then experimentally showed the effect of the temperature change sensitivity [4]. However, at present, this possibility has only been suggested and no specific analysis has been carried out. This paper applies the basic approach of references [1] and [2] to MCF coils to estimate the thermal sensitivity analytically for the first time. As interesting results, the performance difference between the MCF solenoid coil and the SMF symmetrical coil is mentioned. And in the case of MCF symmetric coils, the relationship between the coil parameters (number of cores, number of turns, and number of layers), which can take advantage of MC-FOG in terms of sensitivity to temperature change, is quantitatively clarified in comparison with SMF symmetric coils. In addition, the non-reciprocal phase error of the FIFO element under temperature disturbance is quantitatively clarified. The design policy of the coils proposed from these results is also discussed.

*mitani.shinji@jaxa.jp; phone +81-50-3362-6942; fax +81-29-868-5969; jaxa.jp 


\subsection{Basic theory of non-reciprocal phase error analysis}

When the temperature $T[\mathrm{~K}]$ is time-varying, the interference phase error $\mathrm{d} \phi[\mathrm{rad}]$ per line segment $\mathrm{d} x[\mathrm{~m}]$ at the position $x[\mathrm{~m}]$ of the fiber winding coil is expressed by the following equation (Shupe effect) [1]

$$
\begin{gathered}
\mathrm{d} \phi=\frac{2 \pi}{\lambda}\left[\frac{\delta n}{\delta T}+n \alpha\right] \cdot \tau \cdot \frac{\delta T}{\delta t} d x \\
\tau=\frac{\beta}{\omega}(2 x-L)=\frac{n}{c}(2 x-L)
\end{gathered}
$$

where $\lambda$ : average wavelength [m], $\alpha$ : fiber linear expansion coefficient $[1 / \mathrm{K}], n$ : fiber core refractive index [-], $\delta T / \delta t$ : temperature change rate of $n[1 / \mathrm{K}]$.

$\tau(x)$ is called the diffusion time [s]. $\beta / \omega$ (the ratio of the fiber propagation constant to the optical angular frequency) in $\tau$ is expressed as $n / c$, where $c$ is the speed of light in vacuum $[\mathrm{m} / \mathrm{s}]$ and $c / n$ is the speed of light in the fiber core medium. $L$ is the total length of the fiber [m]. The physical meaning of $\tau$ is the difference in time between the two ends of the fiber when light diffuses from position $x$ to the left and right, respectively. $\tau=0$ when $x=L / 2$ (meaning that the time to reach both ends is equal).

$\delta T / \delta t(x)$ is the rate of temperature change at position $x[\mathrm{~K} / \mathrm{s}]$. The function $\delta T / \delta t(x)$ changes depending on both the heat input to the coil and the way the coil is wound. Given this $\delta T / \delta t(x)$ and integrating over the entire length of the fiber $(x: 0 \rightarrow L)$, we obtain the total amount of interference phase error $\phi$.

$$
\phi=\frac{2 \pi n}{\lambda c}\left[\frac{\delta n}{\delta T}+n \alpha\right] \cdot \int_{0}^{L}(2 x-L) \cdot \frac{\delta T}{\delta t}(x) d x
$$

Since $\tau(x)$ is antisymmetric with respect to $x=L / 2$, if we can give $\delta T / \delta t(x)$ a symmetric form with respect to $x=L / 2$, the integral will be zero. This basic idea is embodied in the symmetric winding of a coil [2].

To convert the phase error $\phi$ into a rate error $\omega_{E}$, we can divide it by the scale factor (Sagnac coefficient) $K_{s}$ [s].

$$
\omega_{E}=\phi / K_{S}
$$

The scale factor $K_{S}$ is given as

$$
K_{S}=\frac{2 \pi L D}{\lambda c}
$$

where $D$ : the average diameter of the coil [m]. However, in this paper, it is not expressed in terms of $\omega_{E}$ or $K_{S}$, but is discussed in terms of the phase error $\phi$ (Eq. (2)) throughout.

\section{PHASE ERROR ANALYSIS APPLIED TO SOLENOID WINDING COIL}

\subsection{SMF coil}

At first, we estimate the phase error $\phi$ when the coil is simply wound from the end (which symmetry winding is not applied) when using the single mode fiber (SMF), not MCF. Following the literature [1], we assume that the temperature change rate $\delta T / \delta t(x)$ over the entire fiber length is distributed as shown in Figure 1. 


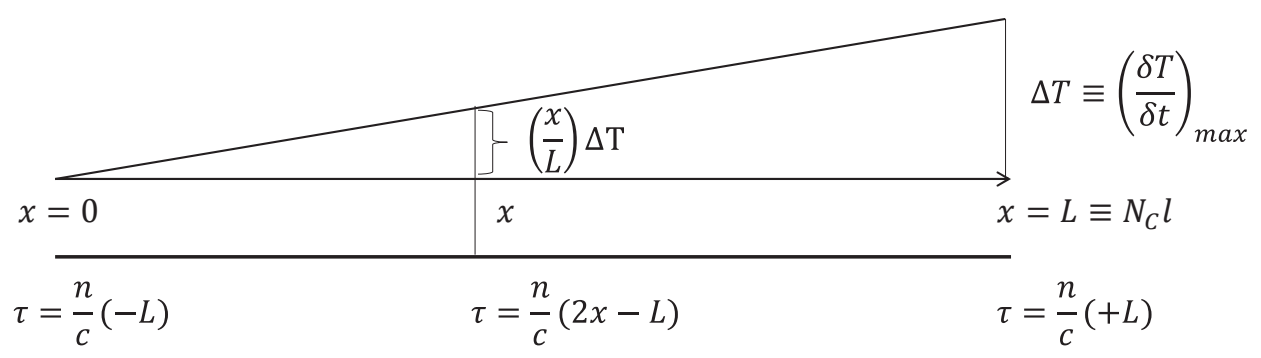

Figure 1. Assumption of the temperature change rate gradient $\delta T / \delta t(x)$ applied to the SMF sensing coil of fiber length $L$. Set the $x$ coordinate axes so that $x=0$ at one end and $x=L$ at the other end ( $L \equiv N_{C} l$ will be required for the subsequent analytical comparison of MCF coils).

In other words, the rate of temperature change is $(\delta T / \delta t)_{\max } \equiv \Delta T$ inside the coil, and 0 outside the coil, and the rate of temperature change is linear in between.

$$
\frac{\delta T}{\delta t}(x)=\frac{x}{L} \cdot \Delta T
$$

Substituting into Eq. (2) and calculating the integral, we obtain

$$
\phi=\frac{2 \pi n}{\lambda c}\left[\frac{\delta n}{\delta T}+n \alpha\right] \cdot \frac{L^{2} \Delta T}{6}
$$

In the literature [2], the rate of temperature change, $\delta T / \delta t(x)$, is assumed to be constant in each layer. In other words, the distribution is assumed to be as Figure 2. Here, $M$ is the number of layers. The fiber length of each layer is $L / M$.

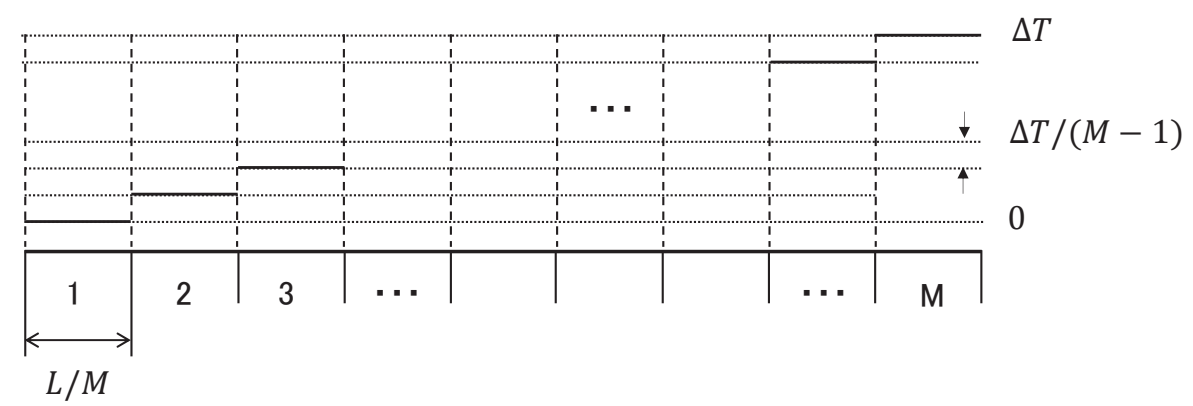

Figure 2. The rate of temperature change, $\delta T / \delta t(x)$, is assumed to be constant in each layer. Under this assumption, the temperature gradient $\delta T / \delta t$ at each coil layer $(x=(\mathrm{L} / \mathrm{M})(\mathrm{j}-1) \sim(L / M) j, j=1, \cdots, M)$ is constant.

Similarly, substituting the above distribution assumption into Eq. (2), we obtain

$$
\phi=\frac{2 \pi n}{\lambda c}\left[\frac{\delta n}{\delta T}+n \alpha\right] \cdot \frac{L^{2} \Delta T}{6} \cdot\left(1+\frac{1}{M}\right)
$$

The difference is only a factor $(1+1 / M)$. The only difference is the coefficient $(1+1 / M)$, and when $M$ is large, there is almost no difference in the values. 


\subsection{MCF coil}

Consider constructing an interference loop with solenoidal windings of MCFs [3]. $l[\mathrm{~m}]$ is the fiber length of MCFs, $N_{C}[-]$ is the number of MCF cores, and $\Delta l[\mathrm{~m}]$ is the splice length between MCF cores (shown in Figure 3). The total length of the interference loop, $L_{T}[\mathrm{~m}]$, is given by

$$
L_{T}=N_{C} l+\left(N_{C}-1\right) \Delta l
$$

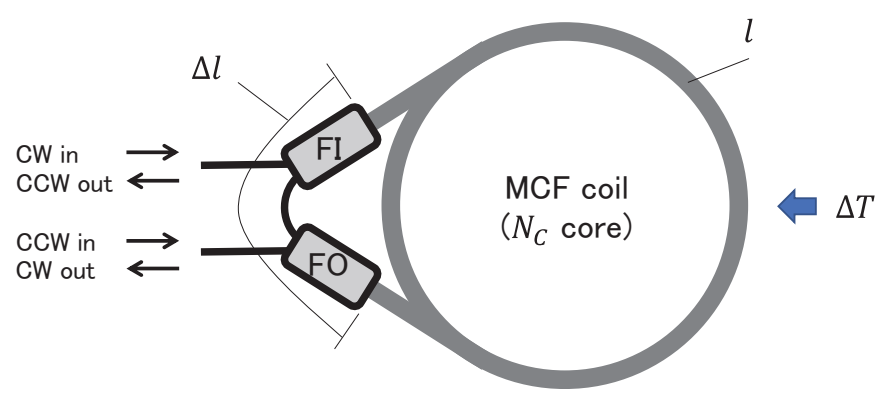

Figure 3. The sensing coil loop architecture constructed with $N_{C}$ core MCF and fan-in (FI)/fan-out (FO) devices. $l[\mathrm{~m}]$ is the fiber length of MCFs and $\Delta l[\mathrm{~m}]$ is the splice length between MCF cores (including the FIFO length).

Figure 4 shows the interference loop stretched out into a single waveguide (for simplicity, the figure shows $N_{C}=3$ ).

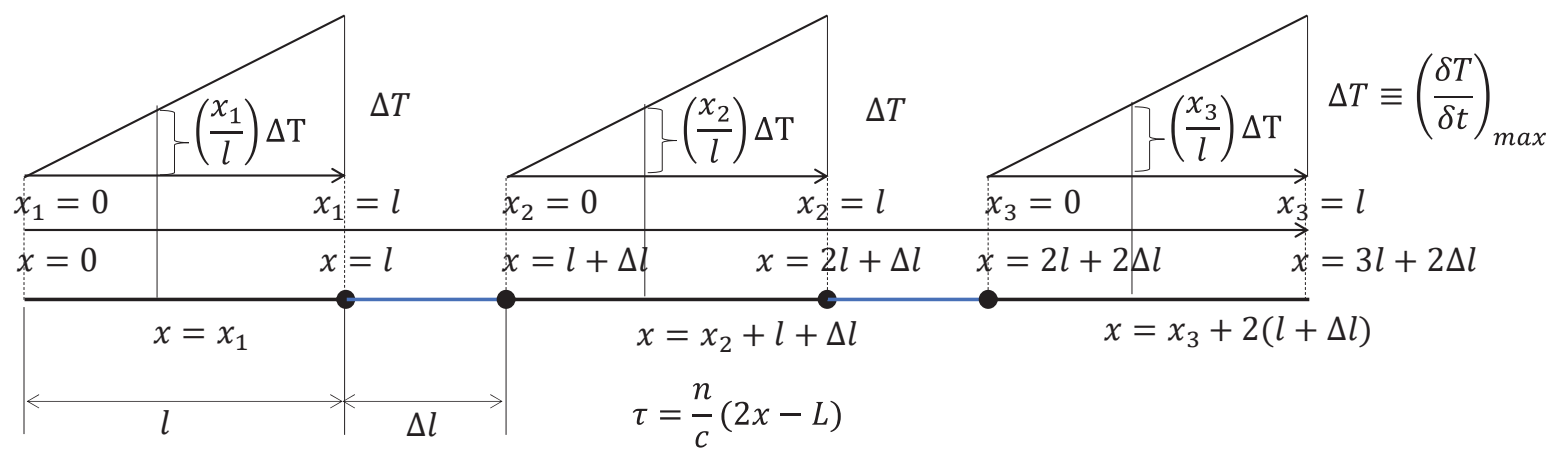

Figure 4. Assumption of the temperature change rate gradient $\delta T / \delta t(x)$ applied to the MCF sensing coil of fiber length $l$ (solenoid winding). Set the $x$ coordinate axes so that $x=0$ at one end and $x=L_{T}$ at the other end.

Consider the case where a temperature distribution is applied to the coil (now assume that there is no temperature change in the SMF of length $\Delta l$ between the MCF cores). Now, let $x_{j}$ be the coordinate of the $j$ th MCF core (in the total length of the MCF fiber) and $x$ be the coordinate of the total length of the interference loop. $x$ and $x_{j}$ are related by

$$
x=x_{j}+(j-1)(l+\Delta l)
$$

The temperature change rate of the $j$ th $\mathrm{MCF}$ core is

$$
\frac{\delta T}{\delta t}\left(x_{j}\right)=\frac{x_{j}}{l} \cdot \Delta T
$$

Substituting to Eq. (2), 


$$
\phi=\frac{2 \pi n}{\lambda c}\left[\frac{\delta n}{\delta T}+n \alpha\right] \cdot \underbrace{\int_{0}^{L}\left(2 x-L_{T}\right) \cdot \frac{\delta T}{\delta t}(x) \cdot d x}_{I}
$$

The integral term $I$ is

$$
I=\sum_{j=1}^{N_{C}}\left[\int_{0}^{l}\left(2 x_{j}+2(j-1)(l+\Delta l)-L_{T}\right) \cdot\left(\frac{x_{j}}{l}\right) \Delta T \cdot d x_{j}\right]
$$

When we calculate this, the term $\Delta l$ vanishes cleanly and we get

$$
I=\frac{N_{C} l^{2}}{6} \cdot \Delta T
$$

Eventually,

$$
\phi=\frac{2 \pi n}{\lambda c}\left[\frac{\delta n}{\delta T}+n \alpha\right] \cdot \frac{N_{C} l^{2}}{6} \cdot \Delta T
$$

\subsection{Comparison between SMF coil and MCF coil}

If we assume that only the fiber length $L$ of the SMF is earned by splicing the MCF of the $N_{C}$ core ( $\Delta l$ is not considered)

$$
L=N_{C} l
$$

Substituting Eq. (15) into Eq. (14), we get

$$
\phi_{M C F}=\frac{2 \pi n}{\lambda c}\left[\frac{\delta n}{\delta T}+n \alpha\right] \cdot \frac{L^{2} \Delta T}{6} \cdot \frac{1}{N_{C}}
$$

Comparing with the solenoid coil of SMF, Eq. (6), we write it down again,

$$
\phi_{S M F}=\frac{2 \pi n}{\lambda c}\left[\frac{\delta n}{\delta T}+n \alpha\right] \cdot \frac{L^{2} \Delta T}{6}
$$

Therefore, it can be said that the phase error of the solenoid coil made of MCF is $N_{C}$ times smaller than that of the solenoid coil made of SMF.

\section{PHASE ERROR ANALYSIS APPLIED TO SYMMETRY WINDING COIL}

\subsection{SMF coil}

From the center $x=L / 2$ of the total length of the SMF fiber, the fiber on the minus side of the fiber coordinate $x$ is called $(-)$, and the fiber on the plus side is called $(+)$. Now, starting from the center of the fiber, we wind the fiber as $(-++-$ $-++-\cdots)$ (called quadrupole (QP) winding), and the number of layers $M$ must be a multiple of 4 , since the total phase of QP winding is canceled by the sum of each of the four layers. Then, the following relationship holds

$$
M=4 P
$$

where $P$ is a positive integer. The distribution of the temperature change rate for this winding method is shown in the figure below. 


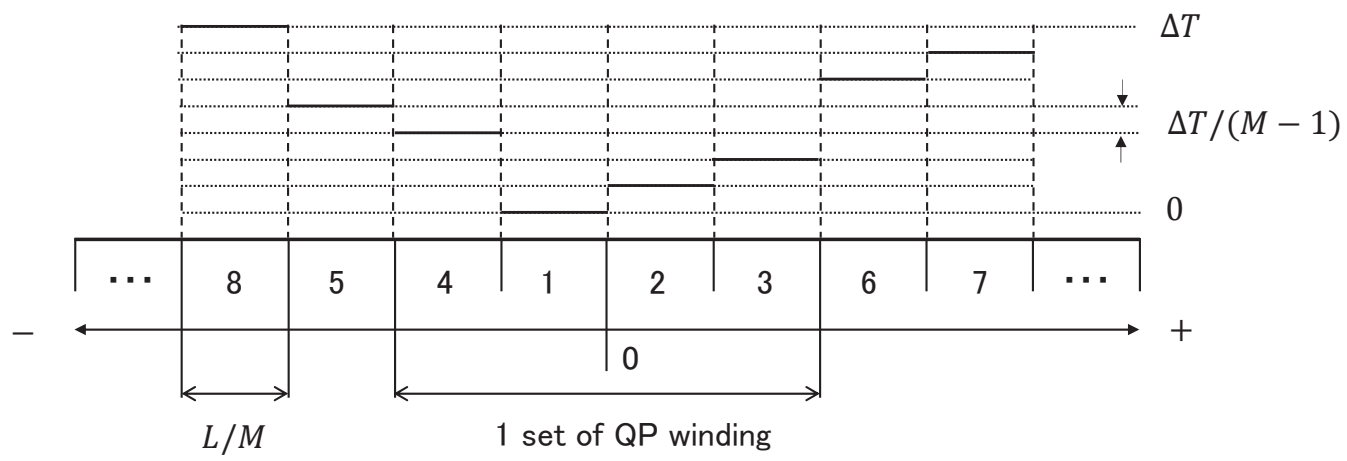

Figure 5. The distribution of the temperature change rate for the QP winding method. Same as Figure 2, The rate of temperature change, $\delta T / \delta t(x)$, is assumed to be constant in each layer.

From Figure 5, the $\delta T / \delta t(x)$ of the $p$ th set $(p=1, \cdots, P)$ and the representative point $x$ of the fiber position in each layer are as follows

$$
\frac{\delta T}{\delta t}(x)=\left\{\begin{array}{c}
4(p-1) \frac{\Delta T}{M-1} \\
\frac{\Delta T}{M-1}+4(p-1) \frac{\Delta T}{M-1} \\
\frac{2 \Delta T}{M-1}+4(p-1) \frac{\Delta T}{M-1} \\
\frac{3 \Delta T}{M-1}+4(p-1) \frac{\Delta T}{M-1}
\end{array} \quad x=\left\{\begin{array}{l}
-\frac{L}{2 M}+\frac{L}{2}-(p-1) \frac{2 L}{M} \\
+\frac{L}{2 M}+\frac{L}{2}+(p-1) \frac{2 L}{M} \\
+\frac{3 L}{2 M}+\frac{L}{2}+(p-1) \frac{2 L}{M} \\
-\frac{3 L}{2 M}+\frac{L}{2}-(p-1) \frac{2 L}{M}
\end{array}\right.\right.
$$

Substituting these values into Eq. (2), the integral term $I$ in the $p$ th set is, respectively,

$$
\begin{gathered}
{\left[4(p-1) \frac{\Delta T}{M-1}\right] \cdot\left[-\frac{L}{M}-4(p-1) \frac{L}{M}\right]} \\
{\left[\{4(p-1)+1\} \frac{\Delta T}{M-1}\right] \cdot\left[\frac{L}{M}+4(p-1) \frac{L}{M}\right]} \\
{\left[\{4(p-1)+2\} \frac{\Delta T}{M-1}\right] \cdot\left[\frac{3 L}{M}+4(p-1) \frac{L}{M}\right]} \\
{\left[\{4(p-1)+3\} \frac{\Delta T}{M-1}\right] \cdot\left[-\frac{3 L}{M}-4(p-1) \frac{L}{M}\right]}
\end{gathered}
$$

The result of the integral term in Eq. (2) is the sum of these (multiplied by the linear element $d x=L / M$ ). If you look closely, you can see that the $4(p-1) \Delta T /(M-1)$ term, the $4(p-1) L / M$ term, and the $[4(p-1)]^{2} L \Delta T / M(M-1)$ term disappear cleanly, even without expanding the calculation. In other words, we only need to pick up the terms that do not depend on $4(p-1)$. Now we can add $P$ sets.

$$
I=\sum_{p=1}^{P} \frac{-2 L \Delta T}{M(M-1)} \cdot \frac{L}{M}=\frac{-L^{2} \Delta T}{2 M(M-1)}
$$

Putting the integral term Eq. (19) back into Eq. (2), we get 


$$
\phi=\frac{2 \pi n}{\lambda c}\left[\frac{\delta n}{\delta T}+n \alpha\right] \cdot \frac{\left(-L^{2} \Delta T\right)}{2 M(M-1)}
$$

Compared to Eq. (6) in which the solenoid winding is applied, it is smaller by an order of $M(M-1)$ (The results in this subsection follow the method in [2], and are naturally consistent with the results in [2]).

\subsection{MCF coil}

Consider an interference loop with QP windings of MCFs, where the length of the MCF fiber is $l$ [m], the number of MCF cores is $N_{C}$ [-], and the connection length between MCF cores is $\Delta l[\mathrm{~m}]$. The total length of the interference loop, $L_{T}[\mathrm{~m}]$, is given by

$$
L_{T}=N_{C} l+\left(N_{C}-1\right) \Delta l
$$

The number of layers, $m$, must be a multiple of 4 . If all layers consist of $P$ sets, then the following relationship holds.

$$
m=4 P
$$

Figure 6 shows the interference loop stretched into a single waveguide (for simplicity, $N_{C}=3$; for odd $N_{C}$, the center of the $\left(N_{C}+1\right) / 2$ th core waveguide is the center of the whole interference loop, as shown in the figure below; for even $N_{C}$, the center of $\Delta l$ between the $N_{C} / 2$ th and $\left(N_{C} / 2+1\right)$ th core waveguides is the center of the whole interference loop. (However, the classification of even/odd $N_{C}$ is not necessary in the following discussion.

\section{the center of the interferometric loop}

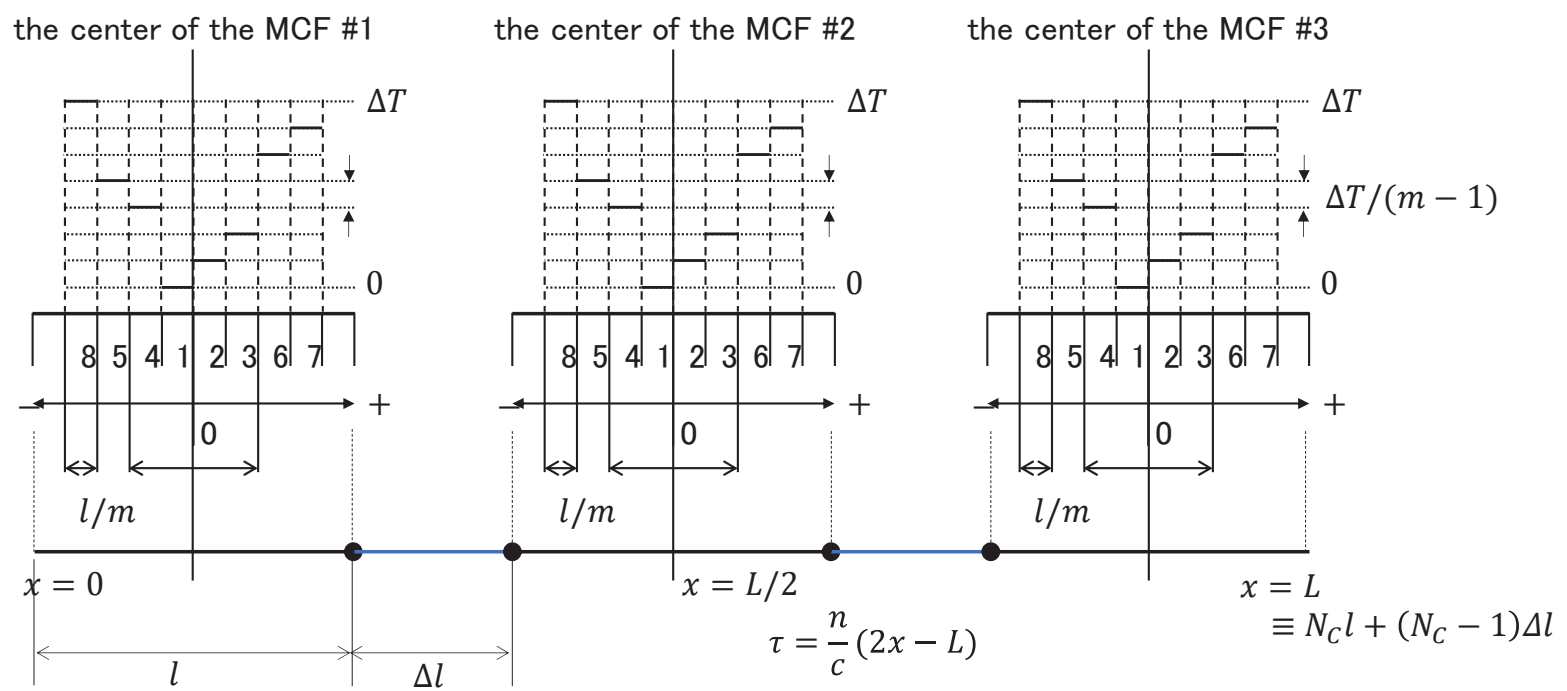

Figure 6. Assumption of the temperature change rate gradient $\delta T / \delta t(x)$ applied to the MCF sensing coil of fiber length $l$ (QP winding). Set the $x$ coordinate axes so that $x=0$ at one end and $x=L_{T}$ at the other end.

Consider the case when a temperature distribution is applied to the coil (now assume that there is no temperature change in the SMF of length $\Delta l$ between MCF cores). 
From Figure 6, the $\delta T / \delta t(x)$ of the $k$ th $\left(k=1, \cdots, N_{C}\right)$ MCF core waveguide and the $p$ th set $(p=1, \cdots, P)$ and the representative point $x$ of the fiber position in each layer are as shown in the following equation.

$$
\frac{\delta T}{\delta t}(x)=\left\{\begin{array}{l}
4(p-1) \frac{\Delta T}{m-1} \\
\frac{\Delta T}{m-1}+4(p-1) \frac{\Delta T}{m-1} \\
\frac{2 \Delta T}{m-1}+4(p-1) \frac{\Delta T}{m-1} \\
\frac{3 \Delta T}{m-1}+4(p-1) \frac{\Delta T}{m-1}
\end{array} \quad x=\left\{\begin{array}{l}
-\frac{l}{2 m}+\frac{l}{2}-(p-1) \frac{2 l}{m}+(k-1)(l+\Delta l) \\
+\frac{l}{2 m}+\frac{l}{2}+(p-1) \frac{2 l}{m}+(k-1)(l+\Delta l) \\
+\frac{3 l}{2 m}+\frac{l}{2}+(p-1) \frac{2 l}{m}+(k-1)(l+\Delta l) \\
-\frac{3 l}{2 m}+\frac{l}{2}-(p-1) \frac{2 l}{m}+(k-1)(l+\Delta l)
\end{array}\right.\right.
$$

Substituting these values to Eq. (11), then, in the $k$ th MCF core waveguide, the integral term $I$ of the $p$ th set is

$$
\begin{gathered}
{\left[4(p-1) \frac{\Delta T}{m-1}\right] \cdot\left[-\frac{l}{m}-4(p-1) \frac{l}{m}+\left\{2(k-1)-\left(N_{C}-1\right)\right\}(l+\Delta l)\right]} \\
{\left[\{4(p-1)+1\} \frac{\Delta T}{m-1}\right] \cdot\left[\frac{l}{m}+4(p-1) \frac{l}{m}+\left\{2(k-1)-\left(N_{C}-1\right)\right\}(l+\Delta l)\right]} \\
{\left[\{4(p-1)+2\} \frac{\Delta T}{m-1}\right] \cdot\left[\frac{3 l}{m}+4(p-1) \frac{l}{m}+\left\{2(k-1)-\left(N_{C}-1\right)\right\}(l+\Delta l)\right]} \\
{\left[\{4(p-1)+3\} \frac{\Delta T}{m-1}\right] \cdot\left[-\frac{3 l}{m}-4(p-1) \frac{l}{m}+\left\{2(k-1)-\left(N_{C}-1\right)\right\}(l+\Delta l)\right]}
\end{gathered}
$$

These sums are multiplied by the linear element $d x=l / m$. Comparing with the SMF QP winding, we can see that only the term $\left\{2(k-1)-\left(N_{C}-1\right)\right\}(l+\Delta l)$ is added. If we sum up this additional term with $k=1, \cdots, N_{C}$ first, we get

$$
\sum_{k=1}^{N_{C}}\left\{2(k-1)-\left(N_{C}-1\right)\right\}(l+\Delta l)=\left\{N_{C}\left(N_{C}-1\right)-N_{C}\left(N_{C}-1\right)\right\}(l+\Delta l)=0
$$

In this way, it disappears completely. The rest of the terms are exactly same as for the SMF QP winding, with the addition of the $N_{C}$ core and $P$ sets.

$$
I=\sum_{k=1}^{N_{C}} \sum_{p=1}^{P} \frac{-2 l \Delta T}{m(m-1)} \cdot \frac{l}{m}=\frac{-N_{C} l^{2} \Delta T}{2 m(m-1)}
$$

Putting Eq. (23) back into equation (11), we get

$$
\phi=\frac{2 \pi n}{\lambda c}\left[\frac{\delta n}{\delta T}+n \alpha\right] \cdot \frac{\left(-N_{C} l^{2} \Delta T\right)}{2 m(m-1)}
$$

\subsection{Comparison between SMF coil and MCF coil}

If we assume that only the fiber length $L$ of the SMF is earned by splicing the MCF of the $N_{C}$ core ( $\Delta l$ is not considered)

$$
L=N_{C} l
$$

Substituting this into equation (24), we get 


$$
\phi_{M C F}=\frac{2 \pi n}{\lambda c}\left[\frac{\delta n}{\delta T}+n \alpha\right] \cdot \frac{\left(-L^{2} \Delta T\right)}{2 m(m-1)} \cdot \frac{1}{N_{C}}
$$

Comparing with the SMF QP coil equation (20), we write it down again,

$$
\phi_{S M F}=\frac{2 \pi n}{\lambda c}\left[\frac{\delta n}{\delta T}+n \alpha\right] \cdot \frac{\left(-L^{2} \Delta T\right)}{2 M(M-1)}
$$

Therefore, it appears that the phase error is $N_{C}$ times smaller when the QP coil is constructed with MCF than when it is constructed with SMF. Some notes on coil winding design will be given in a later section of the discussion.

\section{PHASE ERROR ANALYSIS APPLIED TO FIFO DEVICE}

\subsection{Analysis results}

Estimate the phase error when a temperature change rate of $\Delta T$ is added to the FI at one end of the MCF coil (denoted by the wire element $\Delta x$ ). The figure below shows the interference loop stretched out into a waveguide of total length $L_{T} \equiv$ $N_{C} l+\left(N_{C}-1\right) \Delta l$ (for simplicity, the figure shows $N_{C}=3$ ).

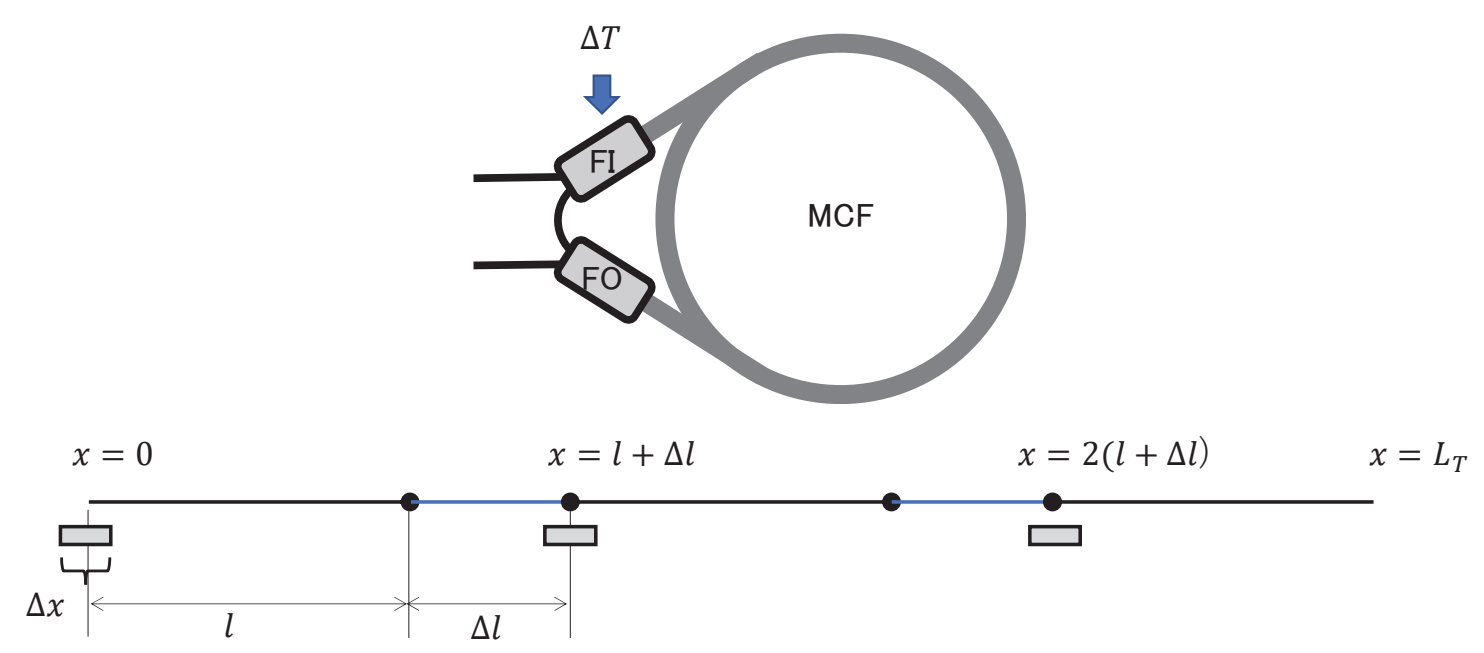

Figure 7. The point on the coil constructed with MCF and FIFO where the rate of temperature change is added (the upper figure). The interference loop stretched out into a waveguide of total length $L_{T} \equiv N_{C} l+\left(N_{C}-1\right) \Delta l$ and FIFO position on the waveguide (the lower figure).

In the interference loop, the FI passes $N_{C}$ times, and the position $x_{k}$ of the $k$ th pass is

$$
x_{k}=(k-1)(l+\Delta l)
$$

Therefore, the phase error can be calculated by adding all the positions of the FI from Eq. (2) to obtain

$$
\phi_{F I}=\frac{2 \pi n}{\lambda c}\left[\frac{\delta n}{\delta T}+n \alpha\right] \cdot \sum_{k=1}^{N_{C}}\left\{2(k-1)(l+\Delta l)-L_{T}\right\} \cdot \Delta T \cdot \Delta x
$$


where the summation part is

$$
\sum_{k=1}^{N_{C}}\left\{2(k-1)(l+\Delta l)-L_{T}\right\}=-N_{C} l
$$

Thus, we have

$$
\phi_{F I}=\frac{2 \pi n}{\lambda c}\left[\frac{\delta n}{\delta T}+n \alpha\right] \cdot\left(-N_{C} l\right) \cdot \Delta T \cdot \Delta x
$$

A similar analysis can be used to estimate the phase error of the line element $\Delta x$ at one end of the SMF coil when a temperature change rate of $\Delta T$ is added. The result is

$$
\phi=\frac{2 \pi n}{\lambda c}\left[\frac{\delta n}{\delta T}+n \alpha\right] \cdot(-L) \cdot \Delta T \cdot \Delta x
$$

This is exactly equivalent to Eq. (29) if the configuration is such that $L=N_{C} l$.

\subsection{Sensitivity compared with silica core fiber}

The FIFO device uses a lens space coupling method in [3,4]. The schematic diagram of the FIFO device is shown in Figure 8. The refractive index of air and the linear expansion coefficient of SUS are used. The air refractive index was calculated by extrapolating the value at a wavelength of $1.56 \mu \mathrm{m}$. The order of the silica core fiber compared to the FIFO device is shown in Table 1. The magnitudes of the $[\delta n / \delta T+n \alpha]$ terms of the two materials are not so different in conclusion, because FIFO is an order of magnitude better in the refractive index temperature change and silica core is an order of magnitude better in the linear expansion coefficient. For space applications, FIFO may have an advantage. This is because the air refractive index temperature change rate is negligible in a vacuum.

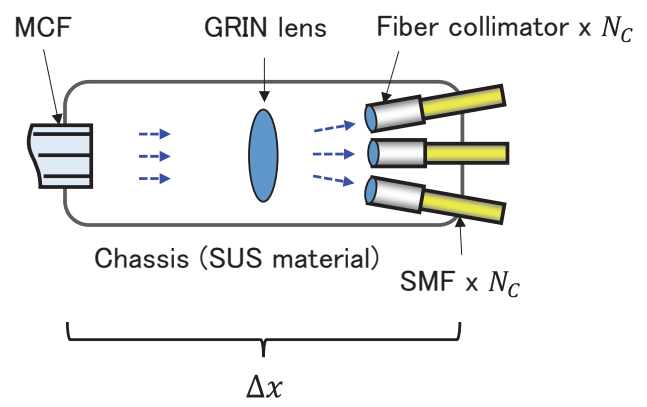

Figure 8. Schematic of the connection using free space optics. This configuration is aimed to separate the optical paths of multiple cores with an aspheric lens and collimate each of them into a single core.

Table 1 . The sensitivity comparison between silica core fiber and FIFO device.

\begin{tabular}{|l|c|r|r|}
\hline \multicolumn{1}{|c|}{ Physical quantity } & Symbol [Unit] & Silica core fiber [1] & \multicolumn{1}{c|}{ FIFO device } \\
\hline Refractive index & $n[-]$ & 1.45 & 1.00 \\
\hline Temperature dependence of $n$ & $\delta n / \delta T[1 / \mathrm{K}]$ & $\sim 10^{-5}$ & $-0.85 \times 10^{-6}$ \\
\hline Linear expansion coefficient & $\alpha[1 / \mathrm{K}]$ & $5 \times 10^{-7}$ & $10 \times 10^{-6}$ \\
\hline$[\delta n / \delta T+n \alpha]$ term & {$[\delta n / \delta T+n \alpha][1 / \mathrm{K}]$} & $\sim 1.1 \times 10^{-5}$ & $\sim 0.92 \times 10^{-5}$ \\
\hline
\end{tabular}




\section{DISCUSSIONS}

\subsection{Design policy to take advantage of MCF symmetrical winding}

It is important to note that when MCF is adopted, not only the fiber length 1 but also the number of layers $m$ may become smaller (compared to the number of layers $M$ in $\mathrm{SMF}$ ), supposing that the number of layers, $\mathrm{m}$, is also $1 / N_{C}$ as the fiber length becomes $1 / N_{C}$. This could be the case if the number of turns and the diameter of the coil are not changed. In this case, substituting $m=M / N_{C}$ to Eq. (25), we get

$$
\phi_{M C F}=\frac{2 \pi n}{\lambda c}\left[\frac{\delta n}{\delta T}+n \alpha\right] \cdot \frac{\left(-L^{2} \Delta T\right)}{2 M\left(M-N_{C}\right)} \cdot \mathrm{N}_{\mathrm{C}}
$$

Therefore, the phase error of the QP coil in MCF is $N_{C}$ times worse than that of the QP coil in SMF. To mitigate this aggravation, the number of layers $m$ should not be lowered below $M / \sqrt{N_{C}}$. For example, if you want to achieve the same Shupe sensitivity as a 24-layer SMF coil using a 7-core MCF, use 12 or more (multiples of 4) layers, since $24 / \sqrt{7} \sim 9.07$. If you use 8 or fewer layers, the Shupe sensitivity will be worse than that of an SMF coil.

Of course, to prevent such deterioration completely, the number of layers should not be changed $(m=M)$. If the coil diameter is not changed, the number of turns in each layer can be reduced to $1 / N_{C}$. In this case, the Shupe sensitivity is improved to $1 / N_{C}$. For example, if you want to use a 7 -core MCF coil with $1 / 7$ the Shupe sensitivity of a 24-layer SMF coil, keep the number of layers at 24 . To achieve this, the number of turns should be reduced to $1 / 7$. If the coil had been wound with 140 turns per layer, for example, the number of turns per layer would be 20 .

In this way, although phase error depends on the coil parameters, it is basically difficult to achieve the same phase error reduction with the solenoid winding of MCF coil as with the symmetrical winding of SMF coil. $1 / N_{C}$ of sensitivity can be achieved by using MCF, while of $1 / M(M-1)$ sensitivity can be achieved by using QP symmetrical winding. If you can use a 12-core MCF, the reduction effect is finally the same as that of a QP 4-layer coil. (For the QP8-layer coil, the MCF has 56 cores!)

We believe that those analytical results are reasonable based on the assumptions we made in our calculations. The basic assumption of the Shupe calculation is that the rate of change of temperature is $(\delta T / \delta t)_{\max } \equiv \Delta T$ inside the coil, and 0 outside the coil, with a linear change in between (even if the inside and outside of the coil are reversed, the result will only be a reversal of polarity). Under this assumption, QP winding reduces the Shupe sensitivity (compared to solenoid winding) by the order of $1 / M(M-1)$, or $M$ squared. In other words, in an ideal calculation, the thicker the number of layers, the greater the reduction.

As a result of the calculation, it is found that the QP winding with MCF has a reduction effect on the order of the square of the number of layers $m$. In addition, when the number of layers is the same as that of SMF, MCF has a "further" $1 / N_{C}$ reduction. However, if the number of layers is reduced to $1 / N_{C}$ compared to that of SMF without changing the number of turns in each layer (because the effect is the square of the number of layers), the MCF becomes $N_{C}$ times less sensitive. In other words, while keeping the number of layers as the same as possible, since the fiber length is $1 / N_{C}$, it is better to make the number of turns in each layer $1 / N_{C}$ (assuming the coil diameter is not changed). In other words, the thickness remains the same, resulting in a flat coil.

\subsection{Analytical Application to Octupole Winding}

A similar calculation could be performed for an octupole (OCT) winding configuration. However, when the OCT winding of the SMF coil is calculated in the same way, the phase error $\phi$ becomes "zero" [5]. This is because the assumption of the calculation is to ignore the contribution of the increase in coil diameter by the fiber diameter as the number of layers is increased. Therefore, we must start again with the calculation considering the fiber diameter. The main result, however, is 
expected to be the same, i.e., if the number of layers is the same, the Shupe sensitivity will be $1 / N_{C}$, and it is not advisable to reduce the number of layers (since it will spoil the effect of symmetric winding).

\subsection{Proposal of a method for connecting cores that minimizes the Shupe error}

As mentioned in the discussion of octupole winding in the previous subsection, the present analysis does not consider the effect of the thickness of the fiber diameter. In fact, the fiber cross-sectional area is finite, and there can be a spatial distribution of the temperature change rate inside the cross-sectional area as well. As a consideration specific to MCFs with multiple cores in the fiber cross section, we propose here a practical splicing method that can further reduce the temperature change rate.

Schematically written, the optical interference loop of the proposed multicore fiber gyroscope can be regarded as an equivalent optical system such that the interference coil with $N_{C}$ single cores is connected in series as shown in Figure 9 $\left(N_{C}=7\right)$. We find that the same idea of symmetrical winding of a coil can be applied to the method of splicing fiber cores. Here, the transmission cores at the distance of the first single-core coil (C7) along the optical path in the clockwise direction from the center point of the equivalent optical path and the transmission cores at the distance of the first single-core coil (C6) along the optical path in the counterclockwise direction are arranged such that they are adjacent to each other in the multicore fiber. The same arrangement can be done for the second (C5 and C4) and third single-core coils (C3 and C2), along the clockwise and counterclockwise directions, respectively, except for the central core (C1).

The analysis in this paper does not address the quantitative effects of this splicing. To perform the analysis, difficulties arise where the spatial distribution of the fiber torsion must be defined within the coil architecture cross section.

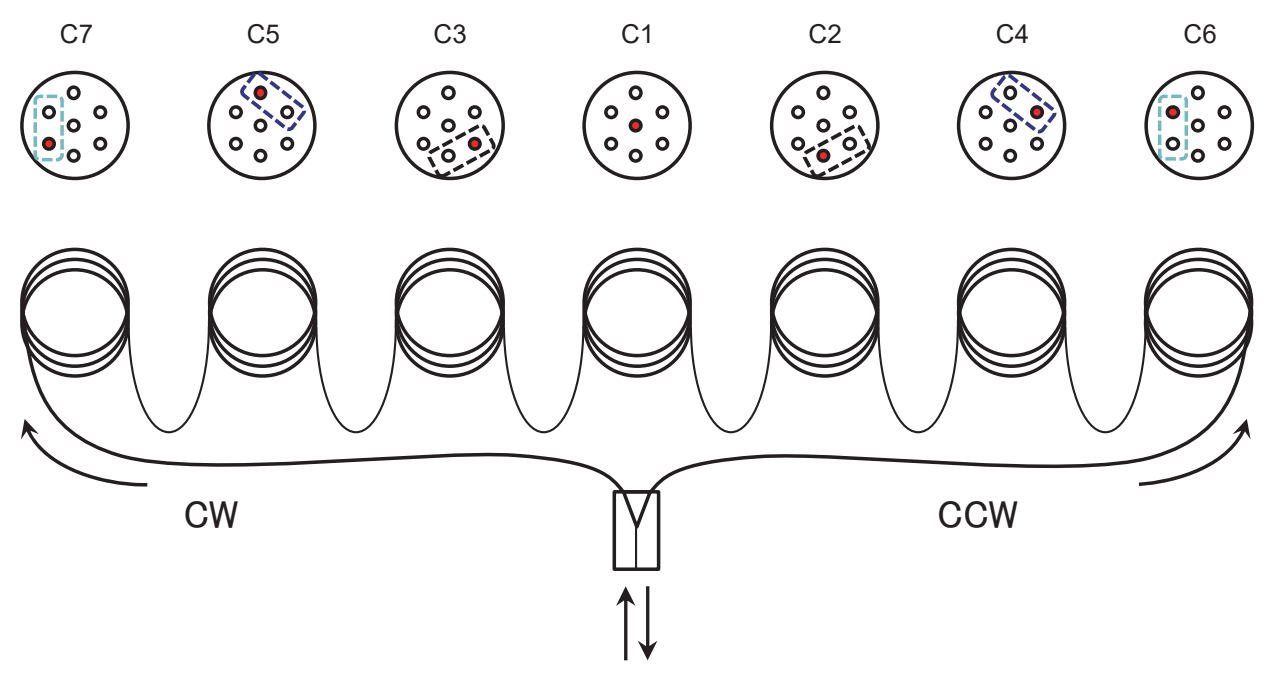

Figure 9. Equivalent optical system to the MCF and FIFO coil. The splicing method between MCF cores is chosen so that the cores (pairs of $(\mathrm{C} 7, \mathrm{C} 6),(\mathrm{C} 5, \mathrm{C} 4)$, and $(\mathrm{C} 3, \mathrm{C} 2))$ that are center symmetric with respect to the center of the equivalent interference loop are spatially close together in the fiber cross section.

\section{CONCLUSIONS}

In this paper, we present the first quantitative analysis of the phase error of a new optical interference coil composed of $\mathrm{MCF}$ and FIFO. The results of the analysis are described below (where, the number of MCF cores is $N_{C}$, and the number of coil layers is $M$ ): 
- If a solenoid coil is constructed with MCF, the sensitivity is reduced by $1 / N_{C}$ compared to a solenoid coil constructed with SMF.

- A symmetrically wound coil with MCF reduces the sensitivity by $1 / N_{C}$ compared to a symmetrically wound coil with SMF. However, the number of layers should not be reduced as much as possible. It is desirable to maintain the number of layers and reduce the number of turns in each layer to $1 / N_{C}$.

- In the case of quadrupole winding, if the number of layers is reduced to $1 / \sqrt{ } N_{C}$ or less, the sensitivity will be worse than that of SMF coils.

- The phase error when a rate of temperature change $(\Delta T)$ is applied to the FI (FO) device has the same effect as when $\Delta T$ is applied to one end of the SMF coil (if the same $\Delta T$ is applied to the FI and FO devices, the effects cancel out).

In conclusion, besides lowering the manufacturing cost of the coil due to the shorter fiber length, the proposed multi-core fiber coil was found to further reduce the phase error sensitivity compared to a single-mode coil of equivalent length, quantitatively demonstrating that the coil is more robust under the temperature changing environment.

\section{ACKNOWLEDGEMENTS}

The authors are grateful to S. Nakamura (Optoquest Co. Ltd.) and K. Nigo (JAXA) for lending their expertise on fiber optic gyroscope and laser optics through useful discussions.

\section{REFERENCES}

[1] D. Shupe, "Thermally Induced Nonreciprocity in the Fiber-Optic Interferometer", Applied Optics, Vol. 19, No. 5 , p. 654, 1980.

[2] N. Frigo, "Compensation of Linear Sources of Nonreciprocity in Sagnac Interferometers", Proc. of SPIE, Vol. 412, p. 268, 1983.

[3] S. Mitani, et. al., "Interferometric Fiber-Optic Gyroscope Using Multi-Core Fiber", Journal of Lightwave Technology, Vol. 37, No. 21, p. 5525, 2019.

[4] S. Mitani, et. al., "Interferometric multi-core fiber optic gyroscope under temperature changing environment", International Conference on Space Optics-ICSO 2018, Chania, Greece, 2018.

[5] S. Mitani, et. al., "Current status of fiber optic gyro efforts for space applications in Japan", Proc. SPIE 9852, Fiber Optic Sensors and Applications XIII, 985208, 2016. 